$\mathbb{P}$ periodica polytechnica

\author{
Transportation Engineering \\ $39 / 2(2011) 69475$ \\ doi: 10.3311/pp.tr.2011-2.04 \\ web: http://www.pp.bme.hu/tr \\ (c) Periodica Polytechnica 2011
}

RESEARCH ARTICLE

\section{Considering predicted road conditions in platoon control design}

\author{
Balázs Németh / Péter Gáspár
}

Received 2010-09-07

\begin{abstract}
The paper focuses on the design of a platoon control system which takes into consideration the safe travel by using the string stability theorem and the knowledge of the inclinations of the road along the route of the platoon. The controlled system incorporates the brake and the traction forces. By choosing the velocity of the platoon fitting in which the inclinations of the road the number of unnecessary accelerations and brakes can be reduced, thus so can the operations of the actuators of the vehicles, i.e. the powertrain and the brake system. The longitudinal dynamics of the vehicles is formulated in a linear controloriented model. In the model the non-linear performance of the predicted road inclinations is considered together with the safety requirements based on the string stability. The control system is designed by using $L P V / H_{\infty}$ control theory.
\end{abstract}

\section{Keywords}

active/semi-active suspension · road-friendly vehicle · passenger comfort $\cdot$ optimal control $\cdot$ weighting function

\section{Acknowledgement}

The work is connected to the scientific program of the 'Development of quality-oriented and harmonized $R+D+I$ strategy and functional model at BME' project. This project is supported by the New Széchenyi Plan (Project ID: TÁMOP4.2.1/B-09/1/KMR-2010-0002) and by the Hungarian Scientific Research Fund (OTKA) through grant No. CNK-78168 which are gratefully acknowledged.

\section{Balázs Németh}

Department of Control and Transport Automation, BME, H-1111 Budapest, Bertalan L. u. 2, Hungary

e-mail: nemeth.balazs@mail.bme.hu

\section{Péter Gáspár}

Systems and Control Laboratory, Computer and Automation Research Institute, H-1111 Budapest, Kende u. 13-17, Hungary

e-mail: gaspar@sztaki.hu

\section{Introduction}

In the paper a method is developed in which saving energy and fuel consumption are taken into consideration in the platoon systems. These requirements are important environmental and economic factors on the roads.

The controllers applied in current adaptive cruise control systems are able to take into consideration only instantaneous effects of road conditions, since they do not have information about the oncoming road sections. In the paper predicted road inclination is considered in the design of the longitudinal control force. The aim in this calculation is to achieve a control force which is similar to the driver's requirement. The driver is usually able to perceive the road conditions, such as the slope of the road ahead, with his eyes.

For example before the downhill slope the driver can see the change in the curve of the road. Here the velocity of the vehicle increases, thus the control force of the vehicle before the slope can be reduced. As a result at the beginning of the slope the velocity of the vehicle decreases, thus it will increase from a lower value. Consequently, the brake system can be activated later or it is not necessary to activate at all. If the velocity in the next road section changes it is possible to set the adequate control force. In the knowledge of the predicted velocity regulations it is also possible to save energy. Moreover, the section of the road, where a speed limit is imposed, different strategies can be considered. Before the regulated section the velocity can be reduced, therefore less energy is necessary for the vehicle. Using the idea of slope and velocity regulation fuel consumption and energy required by the actuators can be reduced.

This idea can be extended to a platoon system, since the method can be applied in the leader vehicle. Here for safety reasons the interaction between the members is necessary to consider and the string stability of the platoon must be guaranteed, see e.g. [7, 8]. In this case the collision of the members of the platoon can be avoided and the spacing error does not increase from vehicle to vehicle in a platoon. Energy consumption should be reduced to a minimum by considering predicted road conditions, e.g. the inclination of the road and the speed limit.

The purpose of the paper is to extend string stability with the 
predicted road conditions. It is shown how a string stable platoon control system can be design by taking into consideration the predicted road conditions. For safety reasons the string stability must be guaranteed all the time, and the energy and fuel consumption can be reduced by exploiting the predicted road conditions. The control purposes are extended with an ability of the control system, in which the official speed limits are also guaranteed.

This paper is organized as follows: Section 2 formalizes the prediction of road conditions and presents the estimation of road inclination. Section 3 contains the consideration of road conditions in a platoon system. Section 4 presents the model based $\mathrm{LPV} / \mathcal{H}_{\infty}$ longitudinal control design. Section 5 shows simulation results and the last section summarizes the conclusion remarks.

\section{Consideration of predicted road conditions}

In this section the predicted terrain characteristics and official speed limits are formalized in a control-oriented model. First the road ahead of the vehicle is divided into equidistant sections. The rates of the slope of the road and those of the speed limits are assumed to be known at the endpoints of all sections. Second the road sections are qualified by different weights, which have an important role in control design. The appropriate selection of weights creates a balance between the velocity of the vehicle and the effect of the relevant slope of the road.

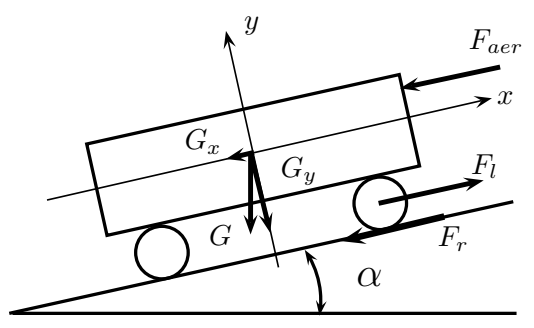

Fig. 1. Simplified vehicle model

The simplified model of the longitudinal dynamics of the vehicle is shown in Fig. 1. The longitudinal movement of the vehicle is influenced by the traction force $F_{l}$ as the control signal and disturbances $F_{d}$. The disturbance forces consist of the rolling resistance $F_{r}$, the aerodynamic force $F_{a e r}$ and force resistance from road slope $G_{x}$ such as $F_{d}=F_{r}+F_{a e r}+G_{x}$. Rolling resistance can be modeled by an empiric formula: $F_{r}=$ $F_{z} f_{0}\left(1+\kappa \dot{\xi}^{2}\right)$, where $F_{z}$ is the vertical load of wheel, $f_{0}$ and $\kappa$ are empiric parameters depending on tyre and road conditions, see [2, 4]. The aerodynamic force can be formulated as: $F_{\text {aer }}=0.5 C_{w} \rho A \dot{\xi}_{\text {rel }}^{2}$, where $C_{w}$ is the drag coefficient, $\rho$ is the density of air, $A$ is the reference area, $\dot{\xi}_{r e l}$ is the velocity of vehicle relative to the air (in case of a lull $\dot{\xi}_{\text {rel }}=\dot{\xi}$, see [2, 4) ).

The acceleration of the vehicle is the following:

$$
\ddot{\xi}=\frac{1}{m} F_{l}-\frac{1}{m} F_{d}
$$

where $m$ is mass of vehicle, $\xi$ is the position of vehicle, and $F_{l}, F_{d}$ are the traction force and the disturbance force, respectively. The predicted course of vehicle can be divided into equidistant sections using $n+1$ number of points (see Fig. 2). The accelerations of vehicle are considered to be constant between these points. In this case the movement of the vehicle using simple kinematic equations is:

$$
s=\dot{\xi}_{0} \frac{\dot{\xi}_{1}-\dot{\xi}_{0}}{\ddot{\xi}}+\frac{\left(\dot{\xi}_{1}-\dot{\xi}_{0}\right)^{2}}{2 \ddot{\xi}}
$$

where $v_{0}=\dot{\xi}_{0}$ is the velocity of vehicle at the first point, $v_{1}=\dot{\xi}_{1}$ is the velocity of vehicle at the second point and $s$ is the distance between these points.

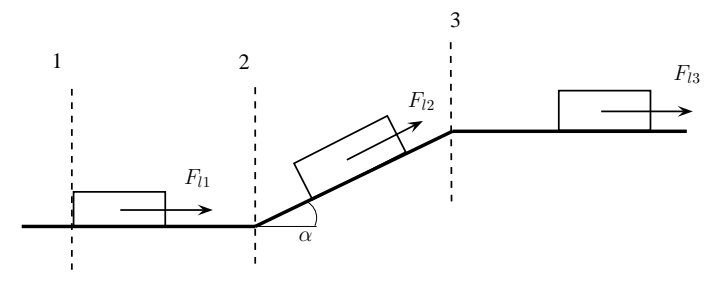

Fig. 2. Division of predicted road

From (2) the following expression is created: $\dot{\xi}_{1}^{2}=\dot{\xi}_{0}^{2}+2 \ddot{\xi} s$. This relationship also applies to the next road section: $\xi_{2}^{2}=$ $\dot{\xi}_{1}^{2}+2 \ddot{\xi}$. Using the dynamic Eq. (1) the next relationship can be formulated:

$$
\dot{\xi}_{2}^{2}=\dot{\xi}_{0}^{2}+\frac{2 s}{m}\left(F_{l 1}-F_{d 1}\right)
$$

where index ${ }_{1}$ shows the point of the first section.

The goal of considering road conditions is to determine a control force, by which the vehicle can drive along its way. At the calculation of the control force it is considered, that the vehicle does not actuate more longitudinal force on its way. Thus, (3) is modified on the next road sections: $\dot{\xi}_{2}^{2}=\dot{\xi}_{0}^{2}-\frac{2 s}{m} F_{d 1}$. The velocities of vehicle are described at each point of the road by using similar expressions:

$$
\begin{gathered}
\left(\dot{\xi}_{3}^{2}=\right) \dot{\xi}_{0}^{2}+\frac{2 s}{m}\left(F_{l 1}-F_{d 1}-F_{d 2}\right)=v_{r e f 2}^{2} \\
\vdots \\
\left(\dot{\xi}_{n+1}^{2}=\right) \dot{\xi}_{0}^{2}+\frac{2 s}{m}\left(F_{l 1}-\sum_{i=1}^{n} F_{d i}\right)=v_{r e f n}^{2}
\end{gathered}
$$

The $F_{d i}$ disturbance force can be divided in two parts: the first part is the force resistance from road slope $F_{d i_{1}}$, while the second part $F_{d i_{2}}$ contains all of the other resistances such as rolling resistance, aerodynamic forces etc. We assume that $F_{d i_{1}}$ is known, however, $F_{d i_{2}}$ is unknown. When the control force $F_{l 1}$ is calculated, only $F_{d 1_{2}}$ influences the vehicle of all of the unmeasured disturbances. In the control design the effects of the unmeasured disturbances $F_{d i_{2}}, i \in\{2, n\}$ are ignored.

It is also an important goal to track the momentary determined reference velocity value. The following equation can also be 
considered : $\dot{\xi}_{0}^{2}=v_{\text {ref } 0}^{2}$. Using the above assumptions the equations of the vehicle at the section points are calculated using (4) in the following way:

$$
\begin{aligned}
\dot{\xi}_{0}^{2}+\frac{2 s}{m} F_{l 1}-\frac{2 s}{m} F_{d 1_{2}} & =v_{r e f 1}^{2}+\frac{2 s}{m} F_{d 1_{1}} \\
\dot{\xi}_{0}^{2}+\frac{2 s}{m} F_{l 1}-\frac{2 s}{m} F_{d 1_{2}} & =v_{r e f 2}^{2}+\frac{2 s}{m}\left(F_{d 1_{1}}+F_{d 2_{1}}\right) \\
\vdots & \\
\dot{\xi}_{0}^{2}+\frac{2 s}{m} F_{l 1}-\frac{2 s}{m} F_{d 1_{2}} & =v_{r e f n}^{2}+\frac{2 s}{m} \sum_{i=1}^{n} F_{d i_{1}} \\
\dot{\xi}_{0}^{2} & =v_{r e f 0}^{2}
\end{aligned}
$$

In the next step weights $\gamma_{1}, \gamma_{2}, \ldots, \gamma_{n}$ are applied to both the predicted reference velocity and the road slope in Eq. (5). An additional weight $Q$ is applied in the last Eqs. (5d). The weights should sum up to one, i.e. $\gamma_{1}+\gamma_{2}+\ldots+\gamma_{n}+Q=1$. While the weights $\gamma_{i}$ represent the rate of the road conditions, weight $Q$ has an essential role, it determines the tracking requirement of the current reference velocity $v_{r e f 0}$. By increasing $Q$ the predicted road conditions become less important. Summarizing the Eqs. (5) and taking the weights into consideration the following formula is yielded:

$$
\begin{aligned}
\dot{\xi}_{0}^{2} & +\frac{2 s(1-Q)}{m} F_{l 1}-\frac{2 s(1-Q)}{m} F_{d 1_{2}}= \\
& =(1-Q)\left(\gamma_{1} v_{r e f 1}^{2}+\ldots+\gamma_{n} v_{r e f n}^{2}\right)+ \\
& +\frac{2 s(1-Q)}{m}\left[F_{d 1_{1}}+F_{d 2_{1}}\left(\gamma_{2}+\ldots+\gamma_{n}\right)+\ldots+F_{d n_{1}} \gamma_{n}\right] \\
& +Q v_{r e f 0}^{2}
\end{aligned}
$$

Based on Eq. (6) the interpretation of the control problem is the following. In order to take the predicted road conditions into consideration in the control design Eq. (6) is applied as a performance of the controlled system. Note that weights have an important role in control design. By making an appropriate selection of the weights the importance of the predicted road condition is considered. For example in case of $Q=1$ and $\gamma_{i}=0, i \in[1, n]$ the control exercise is simplified to a velocity tracking problem without any predicted road conditions. In case of using equivalent weights the predicted road conditions are considered with the same importance. The optimal determination of weights has an important role, i.e. to achieve a balance between the current velocity and the effect of the road slope. Consequently, a balance between the velocity and the economic parameters of the vehicle can be formalized.

Note that in the paper the predicted road conditions are assumed to be known. In Eq. (6) the longitudinal forces depend on the mass of the vehicle and the angle of slope $\alpha_{i}$ : $F_{d l_{i}}=G_{x}=m g \sin \alpha_{i}$ are the forces from the predicted road slopes. A prediction about the slope of the road can be achieved in two ways: either a contour map which contains the level lines is used, or an estimation method is applied. In the former case a map used in other navigation tasks can be extended with slope information, which is applied next time along the same route. Several methods have been proposed for the slope estimation. They use cameras, laser/inertial profilometers, differential GPS or a GPS/INS systems, see [3, 6].

\section{Road conditions in a platoon system}

In this section the control problem is formulated for a platoon system. First the most important theorem of string stability in terms of our method is shown. To meet string stability is an important safety regulation, which must be guaranteed in every vehicle. Then the formula of string stability is integrated with a road condition consideration. The goal is to find an optimal weighting between $\gamma_{1} ; \gamma_{2} ; \ldots ; \gamma_{n}$ and $Q$, by which the string stability can be ensured together with the maximal energy saving.

Several methods for the string stable platoon control have been proposed, see [7]. In this paper the constant spacing control strategy with reference vehicle information is applied in the design of a platoon control system. The tracking requirement is stringent, since every controlled vehicle has to match its position, velocity and acceleration with the vehicle ahead. As a consequence, these strategies require information from the vehicles to guarantee performance specification.

In Section 2 it is shown how the velocity of the vehicle that considers the predicted road conditions is modified. The original reference velocity of the vehicle is $v_{\text {ref } 0}$, while the modified reference velocity is $\dot{\xi}_{0}$ in accordance with Eq. (6). In a platoon system it is necessary to design a string stable controller which meets the predicted road conditions. Eq. (6) can be rearranged in the next form:

$$
\dot{\xi}_{0}^{2}=Q v_{r e f 0}^{2}+(1-Q) M
$$

where $Q$ is the weight and $M$ is a sum of different terms:

$$
\begin{aligned}
M & =\gamma_{1} v_{r e f 1}^{2}+\ldots+\gamma_{n} v_{r e f n}^{2}+ \\
& +\frac{2 s}{m}\left[F_{d 1_{1}}+F_{d 2_{1}}\left(\gamma_{2}+\ldots+\gamma_{n}\right)+\ldots+F_{d n_{1}} \gamma_{n}\right] \\
& +\frac{2 s}{m}\left(F_{l 1}+F_{d 1_{2}}\right)
\end{aligned}
$$

Unfortunately, the relation between the reference velocity and the modified reference velocity is expressed in a nonlinear differential equation form.

In the platoon problem the original reference velocity $v_{\text {ref } 0}$ is the velocity value of the preceding vehicle. Assuming that the difference between the momentary reference velocity and the real velocity is small, the following approximation is used: $\dot{\xi}_{0} \approx v_{\text {ref } 0}$. Applying this relationship (7) is transformed into a linearized form:

$$
\dot{\xi}_{0}=Q v_{r e f 0}+\frac{(1-Q) M}{v_{r e f 0}}
$$

The velocity error between two vehicles shows the efficiency of the controller. For the computation of velocity error it is necessary to analyze the difference between the actual velocity of 
the vehicle $\dot{\psi}$ and the reference velocity. Two kinds of reference velocities are defined: the original $v_{\text {ref }}$ and the modified velocities $\dot{\xi}_{0}$. The derivatives of the error signals are the following:

$$
\begin{aligned}
& \dot{\epsilon}_{i}=\dot{\psi}-v_{r e f 0} \\
& \overline{\dot{\epsilon}}_{i}=\dot{\psi}-\dot{\xi}_{0}
\end{aligned}
$$

Substituting (9) in (10):

$$
\dot{\epsilon}_{i}=\dot{\psi}-Q v_{r e f 0}-\frac{(1-Q) M}{v_{r e f 0}}
$$

Using (11) and (12) the relationship between the two error signals is the following:

$$
\dot{\epsilon}_{i}=T_{i} \overline{\dot{\epsilon}}_{i}
$$

where

$$
T_{i}=\frac{\dot{\psi}-v_{\text {ref } 0}}{\dot{\psi}-Q v_{\text {ref } 0}+\frac{(1-Q) M}{v_{\text {ref } 0}}}
$$

To determine $T_{i}$ it is necessary to measure the actual velocity $\dot{\psi}$ and the original reference velocity $v_{\text {ref } 0}$. Since $M$ is calculated from (8) there is a relationship between $Q$ and $T_{i}$. In the knowledge of $Q$ value it is feasible to calculate $T_{i}$, and vice versa in the knowledge of $T_{i}$ it is feasible to determine $Q$.

In order to take into consideration the predicted road conditions the reference velocity is modified from $v_{\text {ref } 0}$ to $\dot{\xi}_{0}$. Therefore in the computation of the spacing error of the real system is modified:

$\ddot{\epsilon}_{i}+\left(q_{2}+q_{4}\right) \dot{\epsilon}_{i} T_{i}+\left(q_{3}+q_{5}\right) \epsilon_{i}=-q_{1} \ddot{\epsilon}_{i-1}+q_{2} \dot{\epsilon}_{i-1}+q_{3} \epsilon_{i-1}$

The transfer function from the spacing error of $(i-1)^{t h}$ vehicle to $i^{t h}$ vehicle is

$$
H=\frac{\epsilon_{i}}{\epsilon_{i-1}}=\frac{-q_{1} s^{2}+q_{2} s+q_{3}}{s^{2}+\left(q_{2}+q_{4}\right) T_{i} s+\left(q_{3}+q_{5}\right)}
$$

In the next step a robust control is designed. String stability is guaranteed if the infinite-norm of the closed loop system meets the following norm-condition $\|H\|_{\infty}<1$. The construction of the controlled system is shown in Fig. 3. The controlled system tracks the position and velocity of the leader vehicle, and the position and the longitudinal acceleration signal of the preceding vehicle. The velocity of the $(i-1)^{t h}$ preceding vehicle is the same as the original reference velocity of the $i^{\text {th }}$ vehicle, $v_{\text {ref } 0}$. In the method this velocity is modified to $\dot{\xi}_{0}$ by using an $R_{i}$ prefilter, which has an important role in the successful operation of the controller.

The string stability of the platoon system is determined by the $R_{i}$ prefilter. Since string stability is a safety critical requirement, it must be guaranteed in every vehicle in the platoon. Moreover, by considering the predicted road conditions (road slope and velocity regulations) energy can be saved.

It is assumed that there is a controller which guarantees the tracking of the reference signals of the system as accurately as possible.

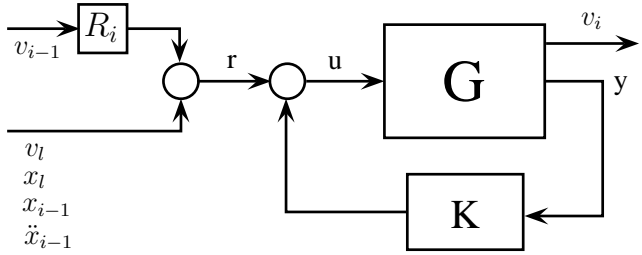

Fig. 3. Block structure of the control system

Eq. 16 expresses that in the knowledge of the controller gains what influence the value of $T_{i}$ has on the transfer function. Consequently, $T_{i}$ influences the infinite-norm of the closed loop system.

The goal of the prefilter design is to find $T_{i}$, which guarantees the string stability of the system and at the same time the predicted road condition is also taken into consideration as much as possible. Since there is a relationship between $T_{i}$ and $Q$ value (see (14)), using the calculated $T_{i}$ the weight $Q$ is calculated. This $Q$ ensures string stability and considers the predicted road condition.

Since the value of prefilter $R_{i}$ changes during the journey of the vehicle, it is necessary to consider it as a varying parameter of the controller. Thus the linear parameter varying (LPV) model is used in the control design. In the next step the robust controller is designed by using the LPV $/ \mathcal{H}_{\infty}$ method.

\section{Longitudinal control design of the platoon system}

The control design is based on the LPV method that uses parameter dependent Lyapunov functions, see [1,5]. This method is based on the possibility of rewriting the plant in a form in which nonlinear terms can be hidden with suitably defined scheduling variables. The LPV modeling approaches allow us to take into consideration the highly nonlinear effects in the state space description in such a way that the model structure is nonlinear in the parameters, but linear in the states. Furthermore this state space representation of the LPV model is valid in the whole operating region of interest. The advantage of LPV methods is that the controller meets robust stability and nominal performance demands in the entire operational interval, since the controller is able to adapt to the current operational conditions.

The longitudinal dynamic equation of the vehicle is transformed into the state-space representation form:

$$
\dot{x}=A x+B_{1} w+B_{2} u
$$

where the state vector of the system $x=\left[\begin{array}{ll}x_{1} & x_{2}\end{array}\right]^{T}$ contains the velocity and the displacement of the vehicle $x_{1}=\dot{\xi}$ and $x_{2}=\xi$, while $u=F_{l}$ is the longitudinal force as a control input and $w=F_{d}$ is the disturbance from the resistances. The components of the state space representation are as follows:

$$
A=\left[\begin{array}{ll}
0 & 0 \\
1 & 0
\end{array}\right], B_{1}=\left[\begin{array}{c}
-1 / m \\
0
\end{array}\right], B_{2}=\left[\begin{array}{c}
1 / m \\
0
\end{array}\right] .
$$

For guaranteeing string stability it is necessary to track the velocity and position of leader vehicle and the velocity, position 
and acceleration of the preceding vehicle. The aim of the servo problem is to ensure that the system output follows these values with an acceptable error. The scheme of the model-based robust $\mathcal{H}_{\infty}$ control design is illustrated in Fig. 4. The disturbances consist of the unknown part of the force disturbances $F_{d i_{2}}$ and the sensor noise. The traction force $F_{l}$ is the difference between the reference signal and the control input $u$.

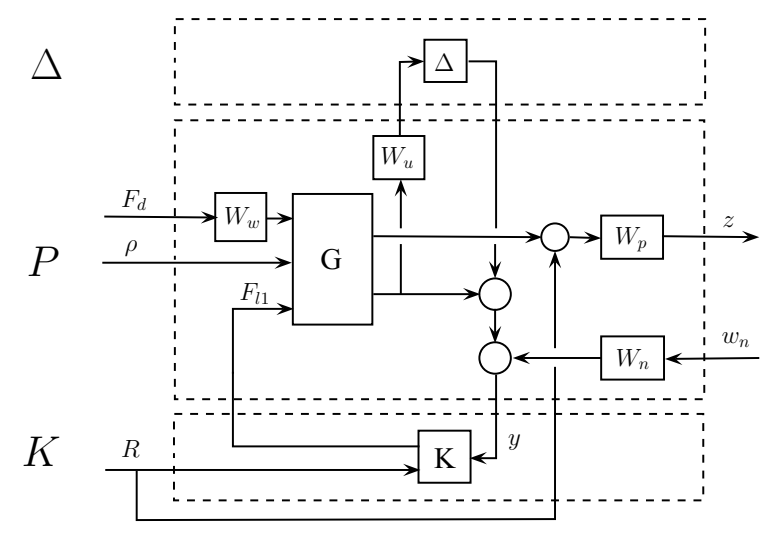

Fig. 4. Closed-loop interconnection structure

In the design of robust control weighting functions are applied. Usually the purpose of weighting function $W_{p}$ is to define the performance specifications in such a way that a trade-off is guaranteed between them. They can be considered as penalty functions, i.e. weights should be large where small signals are desired and small where large performance outputs can be tolerated. The purpose of the weighting functions $W_{w}$ and $W_{n}$ is to reflect the disturbance and sensor noises. $\Delta$ block contains the uncertainties of the system, such as unmodelled dynamics and parameter uncertainty.

In the control problem two performance signals are applied, i.e. $W_{p}=\left[\begin{array}{ll}W_{\text {ref }} & W_{a c t}\end{array}\right]^{T}$. The purpose of weighting function $W_{\text {ref }}$ is to track the reference signal with an acceptable small error. This is important in the low frequencies because the longitudinal dynamics of vehicle causes low frequency dynamics. The purpose of weighting function $W_{a c t}$ is to keep the control input small over the desired operation range. $W_{\text {ref }}$ and $W_{a c t}$ are selected linear proportional forms:

$$
W_{\text {ref }}=\frac{\alpha}{T_{1} s+1}, \quad W_{a c t}=\beta \frac{T_{2} s+1}{T_{3} s+1}
$$

where $\alpha, \beta, T_{1}, T_{2}, T_{3}$ are designed parameters. Similarly, both weighting functions $W_{w}$ and $W_{n}$ are selected in a linear and proportional form. Note that although weighting functions are formalized in the frequency domain, their state-space representation forms are applied in the weighting strategy and in the control design.

Fig. 3 shows that the servo-tracking of the velocity is considered as a performance, which depends on the prefilter of the system $R_{i}$. Thus an LPV controller which is able to handle the changes of the $R_{i}$ is designed. The modified reference velocity can be formulated by using Eq. (6). In Fig. 3 it is shown that the original reference velocity of the preceding vehicle $\left(v_{\text {ref } 0}\right)$ is modified to the reference velocity $\dot{\xi}_{0}$. The relationship between the two velocities is defined by the prefilter $R_{i}$ :

$$
\dot{\xi}_{0}=R_{i} \quad v_{r e f 0}
$$

It shows that the prefilter can be calculated from 20, in which the preceding velocity is measured and $\dot{\xi}_{0}$ is calculated from (6).

Although the control-oriented model is linear, the consideration of predicted road condition makes the model nonlinear. Using this varying parameter the nonlinear model is transformed into the LPV form and the solution of the control design is based on the LPV $/ \mathcal{H}_{\infty}$ method. The linear parameter-varying model is represented by the following state-space representation form:

$$
\dot{x}=A x+B_{w}+B_{2}(\rho) u
$$

where $\rho$ is the gain scheduling parameter, which depends on the prefilter $R_{i}$.

The quadratic LPV performance problem is to choose the parameter-varying controller in such a way that the resulting closed-loop system is quadratically stable and the induced $\mathrm{L}_{2}$ norm from the disturbance and the performances is less than the value $\gamma$. The minimization task is the following:

$$
\inf _{K} \sup _{\varrho \in \mathrm{F}_{\mathrm{P}}} \sup _{\|w\|_{2} \neq 0, w \in \mathrm{L}_{2}} \frac{\|z\|_{2}}{\|w\|_{2}} .
$$

The existence of a controller that solves the quadratic LPV $\gamma$ performance problem can be expressed as the feasibility of a set of Linear Matrix Inequalities (LMIs), which can be solved numerically. The state space representation of the LPV control $K(\varrho)$ can be constructed, see [5].

Finally, the implementation of the designed control system is presented. The schematic structure of the controlled platoon system is shown in Fig. 5. In the paper, the purpose of the control design is to calculate the necessary longitudinal control force. The paper focuses on this upper level controller, in which the LPV method is used. It is assumed that there is another controller which is able to track the control force as a required force. This low-level controller transforms the longitudinal force into a real physical parameter of the actuator. The design of this controller might use more specific techniques that fit the specific nonlinear properties of the actuator.

The platoon has a leader, whose position and velocity are measured, and this information is transmitted to the members of the platoon. Besides these signals the position, velocity and acceleration of every vehicle must be transmitted to the following vehicle in order to ensure string stability. Besides the signals the predicted road conditions are also transmitted (road slope, velocity regulations). From these signals the control system of each vehicle calculates its optimal longitudinal control force.

Since the safe and economical movement of the platoon is determined by the leader vehicle, it is crucial that the leader vehicle uses the predicted road conditions. 


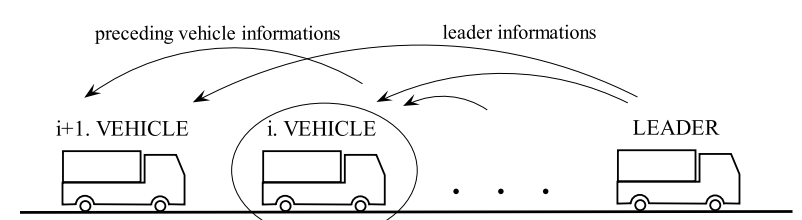

UPPER LEVEL CONTROL

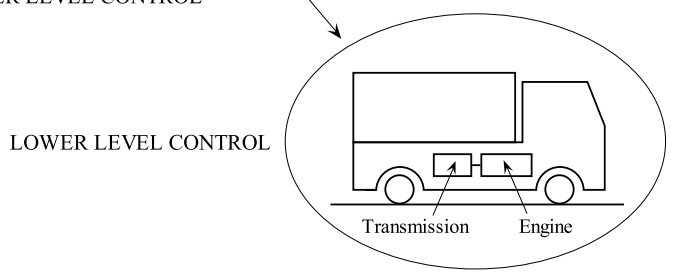

Fig. 5. Implementation of platooning control system

\section{Simulation examples}

In the first simulation the vehicles are traveling along a downhill slope as Fig. 6 shows. In this example three vehicles are used in the platoon. The leader vehicle travels along the downhill slope using the velocity controller, by which the predicted slope can be considered. Therefore the reference velocity of the leader vehicle changes along its course. The other two vehicles of the platoon follow the leader vehicle by using their string stable longitudinal cruise controller.

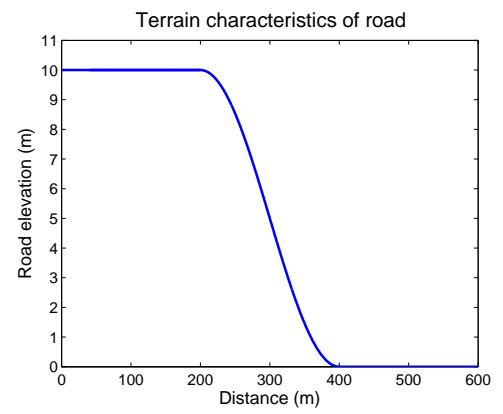

Fig. 6. Terrain characteristics of downhill slope

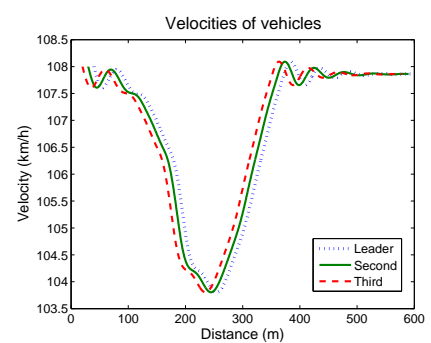

(a) Velocity

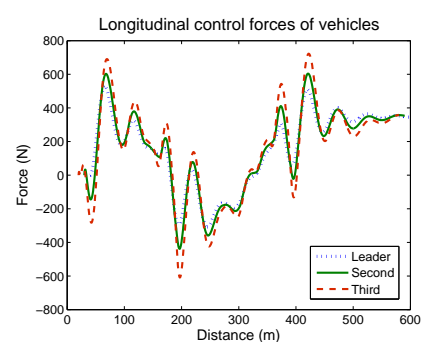

(b) Control force
Fig. 7. Time responses of Controller I on downhill slope

In this simulation the operation of the controller which is designed by using the predicted road conditions (Controller I) is compared to the controller which is designed without road conditions (Controller II). Figs. 7 (a) and 8.a) show that the vehicles track the leader ones with acceptable spacing errors. In both cases the string stability of the platoon systems are guaranteed. In this example the tracking quality does not depend on the ref-

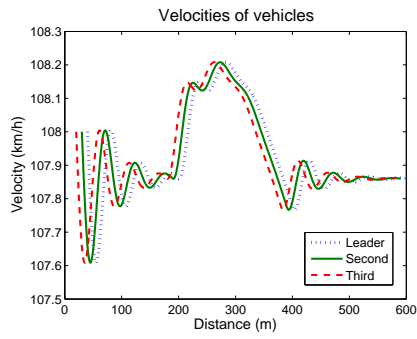

(a) Velocity

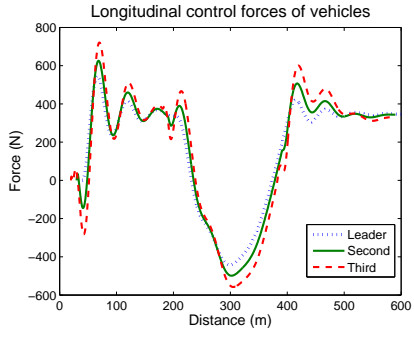

(b) Control force
Fig. 8. Time responses of controller II on downhill slope

erence signal, i.e. the reference velocity could be constant or varying. It shows that the designed robust $\mathrm{LPV} / \mathcal{H}_{\infty}$ controller meets the requirements for vehicle tracking.

Figs. 7. (b) and 8. (b) show the longitudinal forces of vehicles designed in both cases. The difference of the two forces shows the effect of the road slope on the control systems. Controller I generates decreasing control forces in the first part of the downhill slope, thus the longitudinal disturbances are reduced and the necessary traction force to be generated is less compared to Controller II. On the slope Controller I applies less braking force than Controller II, because the platoon using Controller I arrives at the slope at reduced velocity.

In order to qualify the controllers, the necessary absolute values of actuated control energies of the platoon are added up in time. The required energy of vehicles in the platoon using Controller I is $435 k J$, while in the case Controller II it is $559 k J$ The controller which does not take into consideration the road conditions requires $28 \%$ more control energy than the controller proposed in the paper.

The second simulation demonstrates a real traffic case, in which the platoon is arriving at section where a speed limit is imposed, thus the velocity must be reduced. The vehicle is traveling at $81 \mathrm{~km} / \mathrm{h}$ and within $400 \mathrm{~m}$ its velocity must be reduced to $45 \mathrm{~km} / \mathrm{h}$. Fig. 9 shows the simulation of the platoon with the controller, which is based on the predicted road conditions (Controller I). Fig. 9(a) shows that the velocities are reduced continuously before the section with the speed limit. It entails that the velocities near the speed limit sign are lower, therefore less braking energy is necessary after the sign (see Fig. 9(b)).

Fig. 10 shows the simulation results in which the controller does not take into consideration the predicted road conditions (Controller II). In this case the velocities of the vehicles are rapidly reduced (Fig. 10,a)), which requires more control energy (Fig. 10(b)). The sum of actuator energy is lower in case of Controller I $(857 k J)$ than in case of Controller II $(1094 k J)$. These results show that the platoon saves energy at the change of terrain characteristics and at the same time keeps the speed limit. 


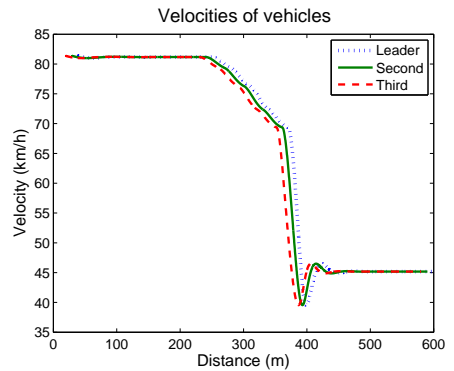

(a) Velocity

Fig. 9. Time responses of controller I in a section with a speed limit imposed

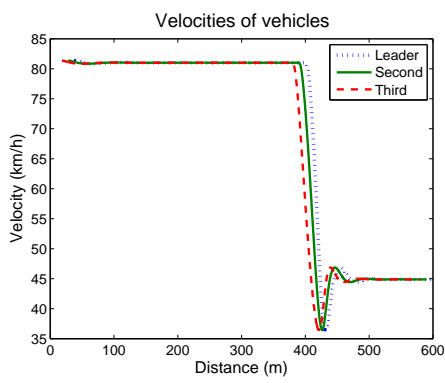

(a) Velocity

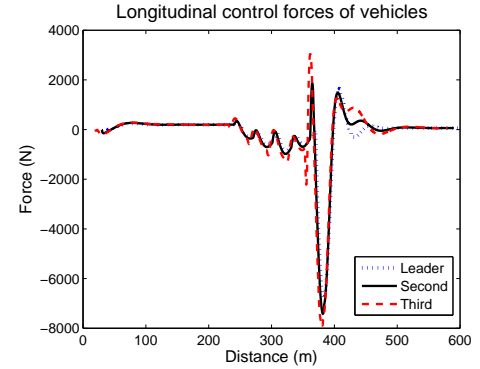

(b) Control force

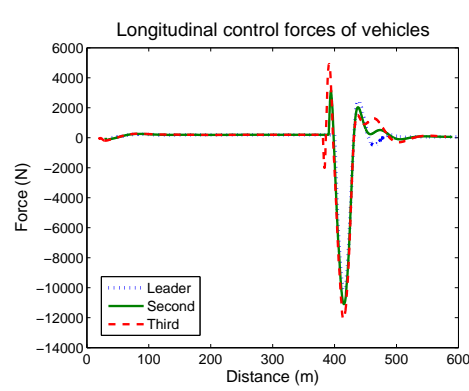

(b) Control force

Fig. 10. Time responses of controller II in a section with a speed limit imposed

\section{Conclusion}

The paper has proposed the design of a platoon control system which combines the string stability theorem and the knowledge of the inclinations of the road along the route. The control design is based on the robust LPV/H $\mathcal{H}_{\infty}$ method, in which both performance specifications and model uncertainties are taken into consideration. Thanks to the integration of vehicle actuators in the platoon and road conditions the simulation results show that the designed control system guarantees string stability and reduces the fuel consumption and the energy required by the actuators. Vehicles save energy at the change of terrain characteristics and at the same time keep compulsory speed limit.

\section{References}

1 Bokor J, Balas G, Linear parameter varying systems: A geometric theory and applications, 16th IFAC World Congress, Prague, 2005.

2 Gillespie T D, Fundamentals of vehicle dynamics, Society of Automotive Engineers Inc (1992).

3 Hahn J O, Rajamani R, You S H, Lee K I, Real-time identification of roadbank angle using differential GPS, IEEE Transactions on Control Systems Technology 12 (2004), 589-599, DOI 10.1109/TCST.2004.825131.

4 Pacejka H B, Tyre and vehicle dynamics, Elsevier Butterworth-Heinemann, 2004.

5 Packard A, Balas G, Theory and application of linear parameter varying control techniques, American Control Conference, Workshop I, 1997.

6 Seo J, Lee J G, Park C G, Bias suppression of GPS measurement in inertial navigation system vertical channel, Position Location and Navigation Symposium (2004), 143-147.

7 Swaroop D, String stability of interconnected systems: An application to platooning in automated highway systems, University of California at Berkeley, 1997. PhD Thesis.

8 Swaroop D, Hedrick J K, String stability of interconnected systems, IEEE Transactions on Automatic Control 41 (1996), 349-357, DOI 10.1109/9.486636. 